Exploring the Effects of Segmentation on Semi-Structured Interview Data with Epistemic Network Analysis

S. Zörgő, Z. Swiecki, A. R. Ruis

This manuscript has been published, please cite it as follows:

Zörgő, S., Swiecki, Z., Ruis, AR. (2021) Exploring Effects of Various Forms of Segmentation on Semi-Structured Interview Data with Epistemic Network Analysis. In: Advances in Quantitative Ethnography. Communications in Computer and Information Science Series. Vol. 1312. Eds. Ruis AR and Lee, SB., pp 78-90. Switzerland: Springer Nature. 


\title{
Exploring the Effects of Segmentation on Semi- Structured Interview Data with Epistemic Network Analysis
}

\author{
$\bowtie$ S. Zörgő1 [0000-0002-6916-2097], Z. Swiecki² [0000-0002-7414-5507], \\ and A. R. Ruis ${ }^{3}$ [0000-0003-1382-4677] \\ ${ }^{1}$ Institute of Behavioral Sciences, Semmelweis University, Budapest, Hungary \\ zorgoszilvialgmail.com \\ ${ }^{2}$ Monash University, Melbourne, Australia \\ ${ }^{3}$ Wisconsin Center for Education Research, University of Wisconsin-Madison, USA
}

\begin{abstract}
Quantitative ethnographic models are typically constructed using qualitative data that has been segmented and coded. While there exist methodological studies that have investigated the effects of changes in coding on model features, the effects of segmentation have received less attention. Our aim was to examine, using a dataset comprised of narratives from semi-structured interviews, the effects of different segmentation decisions on population- and individual-level model features via epistemic network analysis. We found that while segmentation choices may not affect model features overall, the effects on some individual networks can be substantial. This study demonstrates a novel method for exploring and quantifying the impact of segmentation choices on model features.
\end{abstract}

Keywords: Epistemic Network Analysis (ENA) - Semi-structured Interviews · Quantitative Ethnography $(\mathrm{QE}) \cdot$ Methodology $\cdot$ Data Segmentation

\section{Introduction}

Quantitative ethnography $(\mathrm{QE})$ is a nascent field aiming to unify quantitative and qualitative research methodologies in order to facilitate thick description at scale [1]. Most QE studies involve coding raw qualitative data with constructs relevant to the research question(s) and segmenting the data into meaningful parts to explore the interactions of codes [2]. These acts are integral to modelling cognition or behavior and identifying significant patterns. While QE researchers have begun to explore the effects of coding and window length on model features [3-5], the effects of segmentation more broadly have not been similarly examined. In this study, we explored how different approaches to segmentation affect models of patient narratives constructed with Epistemic Network Analysis (ENA), a technique for modelling the structure of connections among codes in qualitative datasets. 


\section{Theory}

Research in $\mathrm{QE}$ is conducted on various kinds of discourse data [2, 6], including log files of online activity, recordings of conversations or interviews, published writing, photographs, field notes, and many others. To facilitate analyses of discourse, QE researchers not only collect or record data that document people thinking, acting, and being in the world, they also transform those data into a representation that makes quantification possible.

This transformation can involve a number of critical decisions. Transcribing video recordings of conversations, for example, might involve: associating spoken sounds with the appropriate word or other verbal expression; identifying which sequences of words should be grouped into utterances; converting utterances into sentences by including punctuation; assigning those sentences to speakers; documenting gestures, facial expressions, or other visual information using consistent notation; applying time stamps; and so on. These processes entail both reducing and standardizing audio-video data into text data for subsequent analysis.

One of the most critical transformation processes that researchers perform is segmentation: the division of data into consistent and meaningful parts. In QE analyses, there are two key levels of segmentation. The first involves segmentation into items or lines (represented as rows in a qualitative data table ${ }^{1}$ ), which are the most elemental units of data. This is the level on which codes are applied, so most researchers choose relatively short segments, such as sentences or paragraphs in formal prose, turns of talk in conversations, or questions and responses in structured interviews. The second level of segmentation involves establishing relational context: groupings of items that are linked for the purposes of interpretation. Defining the recent temporal context-some span of items within temporal or conceptual proximity to one another-is one way of establishing interpretive segmentation. This is the level on which interactions among codes are considered meaningful.

For example, many QE studies use ENA to model the structure of connections among codes in discourse. To operationalize the relational context for each unit of analysis, ENA uses two parameters: conversation and stanza window. Conversations define groupings of items that can be connected in a model. For example, in a dataset that documents the interactions of different project teams on different days, the conversations might be defined by "Team" and "Day." This would group items (say, turns of talk) such that each segment contains all the items from one team's interaction on one day. This means that individuals can make connections only to contributions from their own team, and for a given team, only within a single day's interaction.

Stanza windows define how connection structure is computed within conversations for a given unit of analysis. There are three main types of stanza window:

- Moving stanza windows compute the connection structure of each line in a conversation relative to every line that comes before it within the window. With a moving stanza window of length 4 , for example, each line is connected to the

\footnotetext{
${ }^{1}$ Qualitative data tables contain various kinds of data where rows and columns exhibit ontological consistency. Rows contain the same categories of values (data and metadata), while each column contains one type of information (e.g., age of participants).
} 
three lines that precede it. The connections in each window for which a given unit contributes the referring line (the last line in the window) are then aggregated across all conversations, reflecting the connections contributed by that unit.

- Infinite stanza windows compute the connection structure of each line in a conversation relative to every line that comes before it within the conversation. Thus, the infinite stanza works in the same way as a moving stanza, but there is no limit on the number of previous lines that are included in the window (except for the length of the conversation itself).

- Whole conversation stanzas use the entire conversation as the stanza window, but only connect codes contributed by the same unit. The whole conversation model identifies whether or not a connection occurred in the unit's lines in a given conversation, then aggregates connections across all conversations.

Thus, the operationalization of relational context may have a significant impact on resulting models of connection structure.

Despite the importance of relational context in ENA models, relatively few studies have examined the extent to which different choices of conversation and stanza window affect model features. Ruis and colleagues, for example, found that for one dataset documenting the interactions of engineering student project teams, statistical discrimination between two sub-populations was fairly robust to window length once a minimum length was reached (in their study, that minimum was four lines) [7]. However, they also found that model features and interpretation were more sensitive to window length, and did not stabilize until a window length of seven.

Operationalizing relational context is also particularly challenging with some types of data, e.g. semi-structured interviews, as alternations between interviewer speech and interviewee speech do not necessarily denote meaningful segments for the purpose of analysis. An interviewee may answer one question by addressing various topics relevant to the research objectives in a single turn of talk, which may need to be segmented and analyzed separately. While some researchers choose to parse an interview transcript according to questions and responses when the interview is more clearly structured [8], this may not be a viable option when the interview allows for organic digressions, distal or implicit connections, and the interweaving of several relevant sub-topics. Yet, because many exploratory research initiatives utilize semi-structured interviews for data collection, this form of qualitative data is quite common. Consequently, it is important to understand the effects of different approaches to segmentation on analyses of such discourse.

One approach to assessing the impact of different model parameters - in this case, different conversation and stanza window selections - is through a sensitivity analysis. In a sensitivity analysis, researchers examine whether alteration of one or more parameters invalidates a given inference [9]. In this study, we used a well-studied dataset documenting patient decision-making regarding choice of therapy to explore the effects of segmentation on model outputs. We then used this dataset to address two research questions:

RQ1: Do different segmentations produce significantly different ENA models?

RQ2: Do different segmentations affect the interpretation of individual ENA networks? 


\section{Empirical Data}

\subsection{Study Design and Data Collection}

The empirical data we utilized was derived from a previous study on how patients make decisions regarding their choice of therapy. In that project, we were interested in why patients choose to employ biomedicine only or non-conventional medicine (i.e., Complementary and Alternative Medicine, CAM) either instead of or in addition to biomedicine. The study took place in Budapest, Hungary; data was collected via semi-structured interviews conducted with patients primarily included based on diagnosis group (diabetes, musculoskeletal, digestive, and nervous system diseases). Interviews covered three overarching themes: (i) trusted sources of health-related information, (ii) lay etiology (theories of illness causation), and (iii) patient journey (decisions and experiences). Interviews lasted 60 minutes on average and were sound-recorded and transcribed verbatim. Zörgő \& Peters give a full description of the study, focusing on methodological considerations [10]. We included a total of 26 interviews in the present study; each interview was considered a separate source of data.

\subsection{Coding}

Our deductive code system comprised three levels of abstraction, containing a total of 52 low-level codes. We had three clusters of codes, based on the interview structure: Epistemology (sources of health-related information, appraisal of information; $\mathrm{N}=16$ ), Ontology (concepts of illness and health; metaphors of illness and health; $\mathrm{N}=23$ ), and Behavior (choices of therapy, evaluation of therapeutic efficacy; $N=13$ ). Sentences constituted the lowest level of segmentation (i.e., utterances); coding was performed manually on this level. The analysis below contains the six codes from the Ontology cluster that we used in this study; each code pertains to a lay theory of etiology (see Table 1).

Table 1: Codes capturing patient theories of illness causation (etiology)

\begin{tabular}{|l|l|l|}
\hline \multicolumn{1}{|c|}{ CODE NAME } & \multicolumn{1}{c|}{ LABEL } & \multicolumn{1}{c|}{ DESCRIPTION } \\
\hline $\begin{array}{l}\text { Psychosocial/ } \\
\text { neurological }\end{array}$ & E.e.psych & Emotions, stress, trauma, nerves, nerve damage \\
\hline Psychosocial vitalist & E.e.vital & Energy/qi/prana, block, "law of attraction", spiritual teleology \\
\hline Ecological & E.e.eco & Environmental toxins, chemicals, "electro-smog" \\
\hline Immunological & E.e.immun & Weakness or susceptibility of immune system \\
\hline Nutritional & E.e.nutri & Quality or type of food, additives, toxins in food \\
\hline Genetic & E.e.gene & Inherited illness or susceptibility, genetic causes \\
\hline
\end{tabular}




\section{$4 \quad$ Methods}

\subsection{Constants and Variables of Segmentation}

To explore the effects of segmentation on model features, we used two forms of conversation (source-based and delimiter-based) and three stanza window definitions (whole conversation, infinite stanza, and a moving stanza window of 4 lines). All other model parameters were held constant. Units were defined as individual patients, each of whom was associated with one interview (i.e., one source), and all lines were coded as described above.

\subsection{Forms of Segmentation}

Source-based segmentation. For source-based segmentation, the conversation was defined as one interview transcript, and each source is uniquely associated with one patient. In this type of segmentation, all utterances within an interview were considered relevant context. Source-based segmentation connotes one of the few "naturally" occurring choices for segmenting semi-structured interview data on the conversation level.

Delimiter-based segmentation. For delimiter-based segmentation, the conversation was defined according to a coding process in which two independent raters segmented each source manually to identify shifts in topic within the patient's narrative. This process yielded two versions of the same type of segmentation: DelimiterV1 and DelimiterV2. The former was performed by a research assistant with some prior knowledge of the research topic, the latter by the principal investigator with extensive knowledge of the topic. 


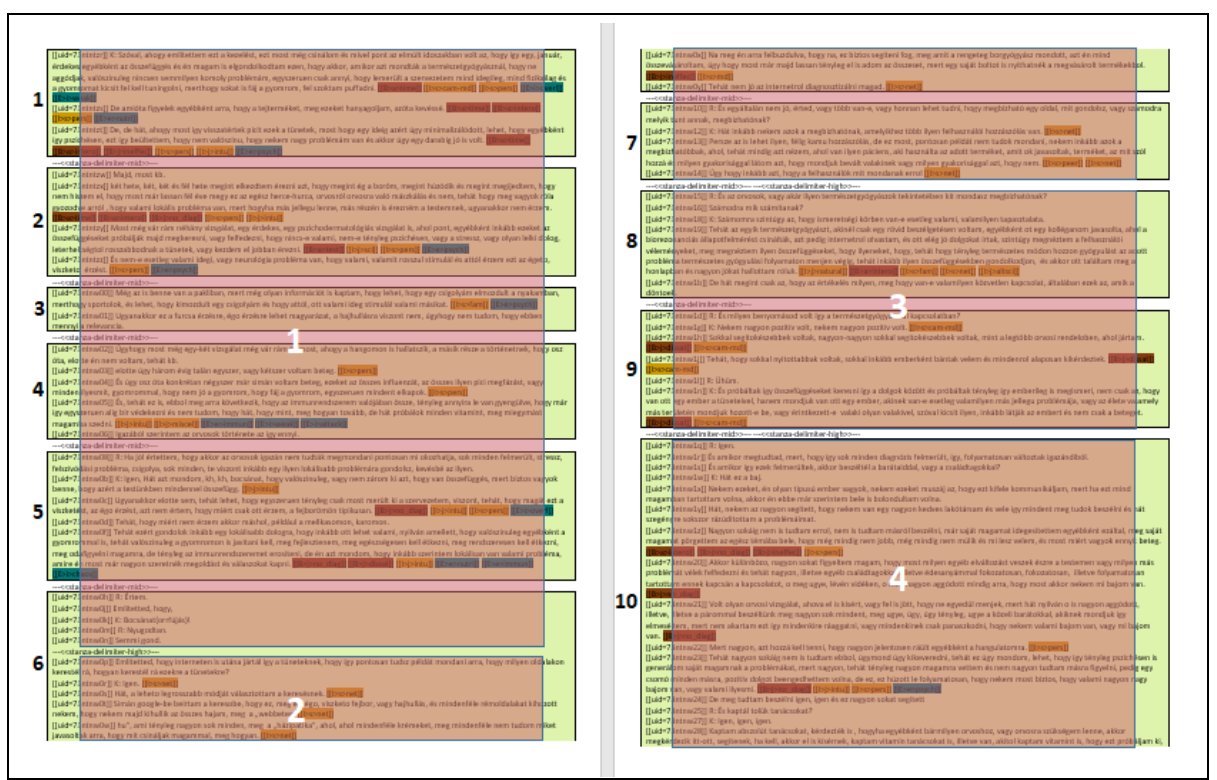

Fig. 1. Delimiter-based segmentation with the same definition of stanza (i.e., recent temporal context) manually performed by two independent raters. The first version (DelimiterV1) is represented by green boxes within the narrative, numbered in black (1-10). The second version (DelimiterV2) is displayed with overlaying red boxes, numbered in white (1-4). Coding is utterancebased (utterance $=$ sentence); codes are depicted within the text representing three code clusters (yellow $=$ epistemology, blue $=$ ontology, red $=$ behavior ).

As Figure 1 shows, although there are some instances when both versions of segmentation (DelimiterV1 and DelimiterV2) agree where a delimiter should occur in the narrative (e.g., after green 7 and red 2), and there are times when the red delimiter is exactly the double of the green (e.g., green 8-9 = red 3), in many cases, delimiting stanzas differ substantially, such as red 1 ending in the middle of green 6 .

\subsection{Model Comparison}

To compare the ENA models constructed with different segmentation parameters, we first constructed a "gold standard" model — that is, the model that aligned best with our qualitative understanding of the data. ${ }^{2}$ Source-based segmentation with an infinite stanza window provided good interpretive alignment with the qualitative data. The

\footnotetext{
${ }^{2}$ The method we describe below can be used to compare any two ENA models that use the same units and codes, and thus does not require a gold standard model. We chose to use one here to reduce the number of comparisons made and simplify the presentation of the results. In many cases it may be difficult to justify a gold standard model. However, if one is justified, an alternate approach is to project all other models into the metric space produced by the gold standard model. Such an approach has the advantage of comparing units of analysis along fewer dimensions, rather than making comparisons in a high-dimensional space. We applied both approaches to these data and our results were consistent between them. Here we present only the highdimensional comparisons to demonstrate the most general approach.
} 
codes pertain to lay etiology, allowing us to model how a patient conceptualizes illness causation. Previous studies have observed that these explanatory models exhibit a high level of intrapersonal congruence, despite the fact that an individual's narrative may contain references to a variety of illnesses. In other words, regardless of how many comorbidities a patient exhibits, from a certain analytical perspective, their theory of illness causation will be consistent or integrated to include all experienced phenomena. Thus, to examine an explanatory model that encompasses the illness with which we included the patient into the study and its comorbidities as well, we chose a segmentation where each individual's narrative is considered a separate, bounded entity within which codes may co-occur, and we regarded every utterance from an individual as closely connected. Code co-occurrences within aggregated interview narratives and mean networks were produced for the two main groups in the study: users of biomedicine and users of CAM.

Table 2: The nine models created to test the effects of segmentation on network structure

\begin{tabular}{|c|c|c|c|}
\hline MODEL & UNIT & CONVERSATION & STANZA WINDOW \\
\hline Gold standard & \multirow{9}{*}{$\begin{array}{l}\text { Individual } \\
\text { (source) }\end{array}$} & \multirow{3}{*}{ Source } & Infinite stanza \\
\hline Model 1 & & & Whole conversation \\
\hline Model 2 & & & Moving window (4) \\
\hline Model 3 & & \multirow{3}{*}{ Source + DelimiterV1 } & Whole conversation \\
\hline Model 4 & & & Infinite stanza \\
\hline Model 5 & & & Moving window (4) \\
\hline Model 6 & & \multirow{3}{*}{ Source + DelimiterV2 } & Whole conversation \\
\hline Model 7 & & & Infinite stanza \\
\hline Model 8 & & & Moving window (4) \\
\hline
\end{tabular}

Following the approach described in [11], we compared each patient's gold standard ENA model to their corresponding model under eight combinations of conversation/stanza window by computing the pairwise correlations (Pearson's $r$ ) between the normalized adjacency vectors (i.e., the vectors representing the normalized connection strengths between each unique pair of codes) of each unit. A high-magnitude correlation between normalized adjacency vectors suggests that the structure of connections associated with each unit was similar in both models. A low-magnitude correlation between normalized adjacency vectors suggests that the models produced different connection structures.

Prior to computing the correlations, individuals with no connections in one of the two models compared in a given correlation were excluded. This situation was relatively rare - only six patients had no connections under one or more of their models.

To evaluate how the networks changed overall for a given comparison, we averaged the correlations. Prior to averaging, we transformed the correlations using Fischer's $Z$ transformation, which allows for meaningful averages of correlation coefficients and the calculation of confidence intervals. To test for significant differences between models, we tested whether the average correlation between models was significantly different from a high correlation value (0.90) using the $95 \%$ confidence intervals. 
To inspect individual differences, we constructed boxplots of the (untransformed) correlation values. This enabled us to explore the extent to which individual networks might change substantially even if the average correlation between two models is high.

Finally, to explore model differences due to segmentation in more detail, we compared ENA network graphs from the gold standard model to network graphs from the other models for individuals whose vectors were substantially different. In these network graphs, the nodes correspond to the codes in the analysis, and the edges reflect the relative frequency of co-occurrence between codes. Thicker and more saturated edges indicate higher relative co-occurrence.

\section{$5 \quad$ Results}

Figure 2 shows the pairwise correlations between the eight models generated with differing segmentation parameters (mod1-8) and the gold standard model (gsmod).

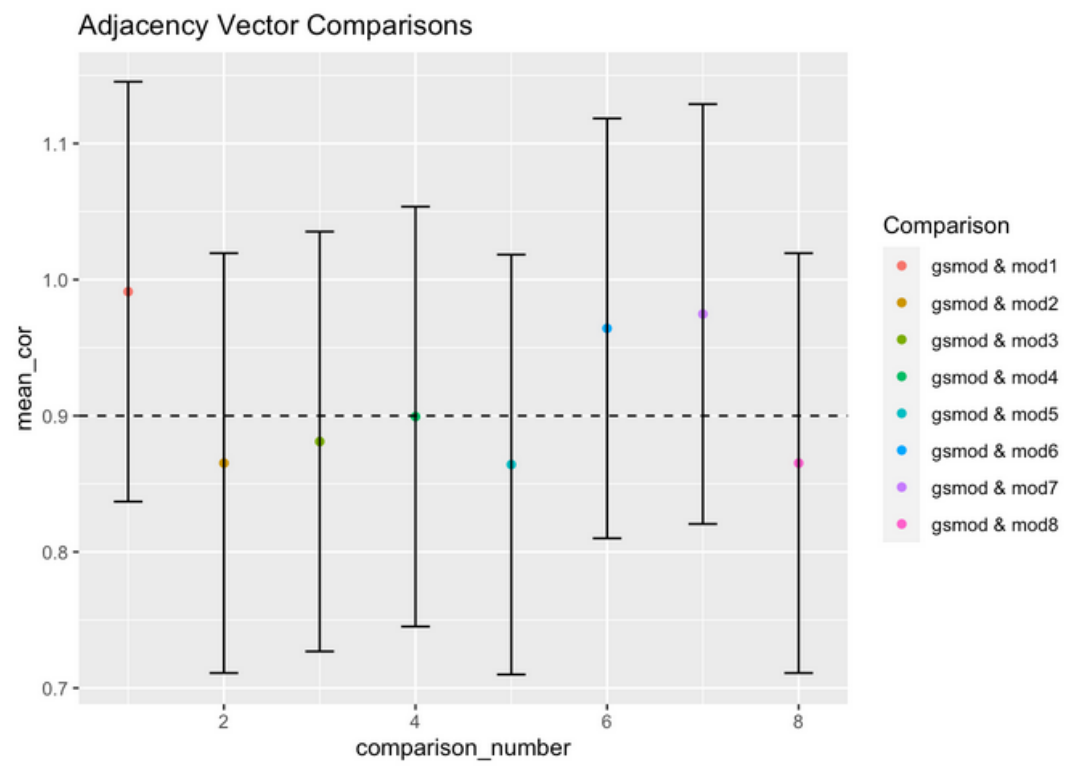

Fig. 2. Mean pairwise correlations (Pearson's r) between the normalized adjacency vectors of models created with different types of segmentation (mod1-8) and the gold standard model (gsmod). The upper bounds of the 95\% confidence intervals for all comparisons are above the critical value of 0.90 , suggesting that the models are highly correlated with the gold standard.

All of the models generated with different segmentations (i.e., different combinations of conversation and stanza window) are highly correlated with the gold standard model (gsmod). That is, for no model is the upper bound of the $95 \%$ confidence interval below the critical value of 0.90 . Furthermore, no model's confidence interval had a lower bound less than 0.70 , indicating that all models were highly correlated with the gold standard model. 
While there are no statistically significant differences between the adjacency vectors of the gold standard model and the other models, some comparisons show that certain individuals changed substantially (see Figure 3). In particular, 97 comparisons yielded correlations below 0.90 , and some are as low as 0.20 . This suggests that while the models as a whole are highly correlated, some individuals have very different networks under models constructed with different segmentations.

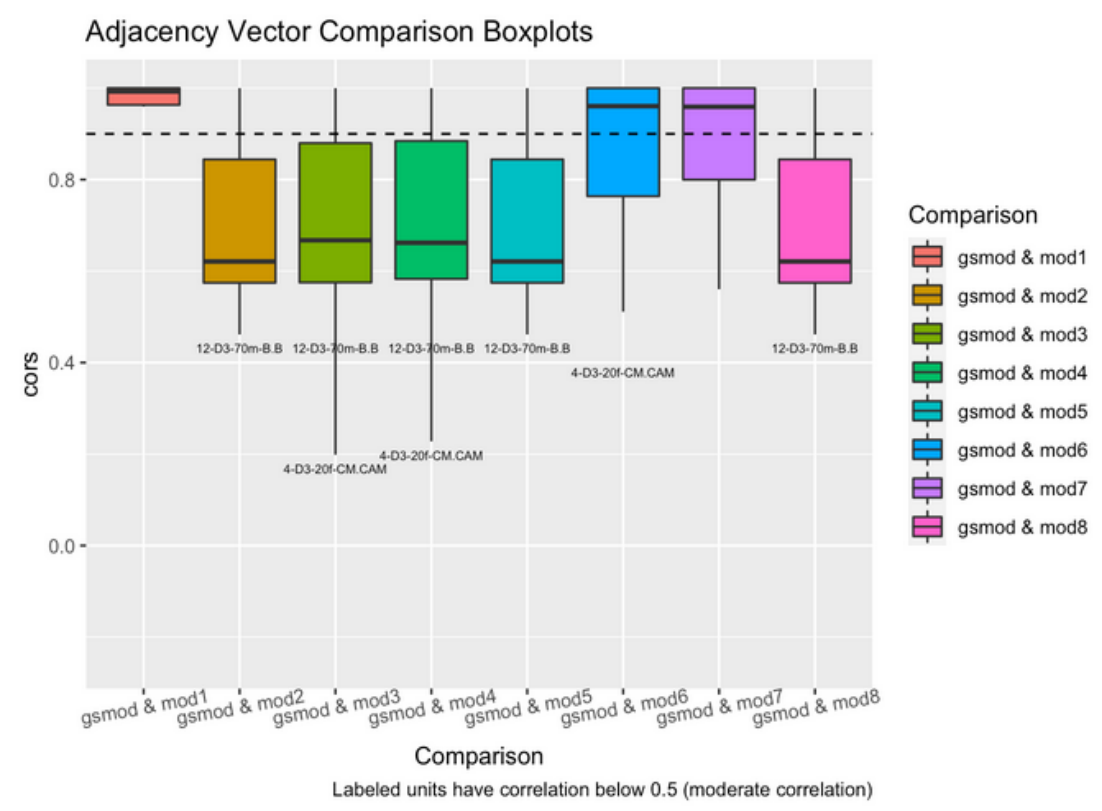

Fig. 3. Box plots showing the distribution of pairwise correlations for each model comparison. Although mean pairwise correlations between models were uniformly high, many individual pairwise correlations were substantially lower.

To explore the extent to which interpretation of patient networks changed due to segmentation, we chose two examples: Patient 25 and Patient 16. Source 25 is the interview transcript of a patient in the CAM group, a female in her twenties, suffering from Crohn's disease and ulcerative colitis; she was diagnosed at the age of 14. Source 16 is the interview transcript of a patient in the biomedicine group, also a female in her twenties, suffering from type 1 diabetes; she was diagnosed at the age of 2 . Both patients require biomedical treatment, but Patient 25 employs complementary therapies as well. Both Crohn's disease and type 1 diabetes are chronic conditions, and in the current biomedical understanding, their causes are unknown. In instances of chronic conditions of unknown origin, lay explanatory models of illness may play an even more significant role in patient decision-making regarding choice of therapy. This was also why these specific patients were selected for closer scrutiny. The source-based moving stanza model of Source 25 exhibited one of the most substantial changes compared to the gold standard, while Source 16's same two models remained very similar. 




Fig. 4. The network models of two patients in two different model comparisons. Unit 25 (top, teal) is an individual in the CAM group, while Unit 16 (bottom, red) belongs to the biomedicine group. The networks were generated using an interview transcript (source) as conversation, and the stanza window was defined as infinite stanza (left) and moving stanza (right). The former is how we defined the gold standard model. The figure illustrates the extent to which a network structure can change with segmentation (Source 25) or remain roughly similar (Source 16), compared to the gold standard.

The graphs in Figure 4 compare the co-occurrence of etiology codes within the narratives of the two individuals. Both network models were generated with source-based segmentation, the graphs on the left with an infinite stanza window (gold standard), the ones on the right with a moving stanza window of 4 utterances. While the basic structure of the biomedical patient's network (bottom models in red) stays consistent in the two versions of segmentation (and all other types of models in our study), the CAM individual's network exhibits substantial differences. In both versions of segmentation, the biomedical patient makes connections among psychological, ecological, and nutritional factors within their theory of illness causation. The CAM patient, on the other hand, makes connections among psychological, vitalist, immunological, and nutritional 
factors in the infinite stanza version of the model, but only a fraction of these remain in the moving stanza version: the connection among psychological, vitalist, and nutritional factors remains (with different edge weights), but the connection to immunological factors disappears, as well as the connection between codes Vital and Nutri.

The first third of Patient 16's discourse does not contain any etiology codes, these only appear in the remainder of the interview; Patient 25's narrative, in contrast, contains etiology codes in the first and second third only. The average distance between two etiology codes in Source 16 is much less than in Source 25 (once every 7.6 lines and once every 40.8 lines, respectively). Compared to the entire length of the interview, the number of utterances spanning the first and last coded utterance is proportionally similar in the two patients' discourse (Source 16: 88 out of 371 utterances, which is $23.7 \%$ of the total number of utterances; Source 25: 451 out of 2126 utterances, which is $21.2 \%$ of the total number of utterances). Thus, etiology codes spanned about the same length in both interviews proportionally, yet there were marked differences in the position of these codes within the interview and relative to each other. These features may explain a unit's sensitivity to changes in model parameters.

Our employed R scripts, disclosed results, and extra visualizations can be openly accessed at our Gitlab repository: https://gitlab.com/szilvia/icqe20-segmentation.

\section{Discussion}

We compared the normalized adjacency vectors of units in eight different ENA models to our gold standard in order to assess the extent to which varying segmentation affects overall network structure. As our results show, different segmentation choices did not produce models with significantly different features in aggregate, but for some individuals, there were substantial differences in their ENA networks under models produced with different segmentations.

These differences in unit sensitivity to changing model parameters may be caused by several factors. Apart from their frequency, the location of codes within the discourse seems to be an influencing factor; codes appearing earlier in an interview, for example, might increase the unit's sensitivity to varying segmentation. Another potentially important factor is code proximity, as relationships between codes in close proximity may look similar in infinite and fixed window models, but more distal codes are less likely to be connected in fixed window models. Further research is needed to more fully understand the factors that contribute to a unit's sensitivity to varying model parameters and to understand what segmentation choices are most appropriate for different kinds of data and research questions.

The source-based infinite stanza and whole conversation models capture the broadest range of possible connections, while the delimiter-based models and source-based model with a moving stanza window of 4 lines capture code co-occurrences taking place in more recent temporal context. Yet, distance between codes (varying number of utterances between two codes) may or may not reflect cognitive proximity in a semistructured interview. In our specific example, an interviewee may talk about one illness at the beginning of a narrative that may receive a set of etiology codes, then go on to 
talk about a comorbidity with another set of etiology codes, and alternate between these throughout the interview. This situation could indeed lead to a larger distance between two etiology codes, but there is a latent variable affecting that distance: the illness to which the codes refer. Thus, it may be beneficial to segment a narrative based on an underlying variable, such as illness in this particular case, and examine the relationship among codes vis-a-vis this type of segmentation. Naturally, other clusters of codes (pertaining to other phenomena or research questions) may be influenced by a different variable. For example, our study also worked with a cluster of codes relating to the patient's trusted sources of information; this cluster may be less meaningful in an illness-based narrative structure and more adequately understood with a discourse segmentation determined by stages in a patient journey (e.g., before diagnosis/after diagnosis or according to various employed treatments).

From a pragmatic point of view, one could assert that each of our nine models (gold standard plus comparisons), highlights a different aspect of our data. In the sourcebased segmentation, the whole conversation model (mod 1) showed us all possible connections among all codes in the given source, but only whether they occurred or not, while the infinite stanza model (gsmod) provided insights into the relative frequency of those connections. Finally, the source-based moving stanza window of 4 model (mod 2) indicated which of those weighted connections were among codes that are spatially/temporally closer to each other within the interview. The delimiter-based models depicted the same basic principles, but within segments of narrative that were manually determined to be closely connected (according to a certain definition of stanza: i.e., topic of discussion). Depending on the analytical goal, all of these models are potentially useful. The method we present here for comparing models with different segmentation parameters allows researchers to assess whether and to what extent any two models are significantly different, but it does not indicate which model is better. That decision remains one that must be based on the research questions being asked and deep qualitative engagement with the data and the context in which they were collected.

Even if segmentation has little effect on model features overall, this study suggests that individual networks may vary substantially under different segmentation parameters, and thus other factors may be important when defining the conversation and stanza window. For example, the (relative) frequency of codes can have a significant impact on model features, as whole conversation models are sensitive only to the presence or absence of a code in the conversation, while infinite stanza windows are also sensitive to how often codes occur and where in the conversation they appear. Additional studies are needed to explore the relationship between segmentation and the (relative) frequency and distribution of codes.

\section{$7 \quad$ Limitations}

Our study had several limitations. First, to reduce the number of comparisons for simplicity of presentation, we selected a gold standard model (source-based, infinite stanza), yet in reality, such "gold standards" do not exist for a given dataset, much less for datasets in general. The method we present here does not require a gold standard- 
it can be used to compare any two models with the same units and codes, and thus has significant advantages over projecting one model into the metric space defined by another model - but all comparisons are still pairwise, and thus there are a large number of possible comparisons. In addition, the lowest correlations do not necessarily reflect networks with the most significant differences (for any two models), as the overall structure of connections could be similar even if all the edge weights are different. Many small changes to the line weights could result in a poorer correlation than one large change (e.g., a connection present in one model and not present in another), but the impact on interpretation may be greater in the latter case. Furthermore, correlating the normalized adjacency vectors is merely one way to compare network structures; other modes of comparison, such as correlating the eigenvectors, might yield different results. Lastly, as discussed above, the frequency of code occurrence may be a significant factor, particularly if code frequencies are low, as many were in this dataset. Further research is needed on the effects of segmentation on datasets with different frequencies and distributions of codes.

\section{$8 \quad$ Closing Remarks}

Our study aimed to investigate the effects of different segmentation parameters on model features, and in particular to explore whether and to what extent different conversation and stanza definitions changed network structure or interpretation. We present a method that can be used to compare any two models with the same units and codes, which can guide model parameterization, but this study also shows that more research is needed to address several key questions: Under what conditions will the choice of segmentation start to have large effects? How do these effects occur? and What influences their magnitude? We believe that inquiry in this domain can aid the construction of more accurate (network) models of qualitative data, which in turn, will facilitate our understanding of human cognition and behavior.

\section{Acknowledgements}

The authors are grateful to collaborator GJY Peters and to research assistants Anna Geröly, Anna Jeney, and Krisztina Veres for their rigorous work in the project that provided our empirical data. This work was funded in part by the National Science Foundation (DRL-1661036, DRL-1713110), the Wisconsin Alumni Research Foundation, and the Office of the Vice Chancellor for Research and Graduate Education at the University of Wisconsin-Madison. The opinions, findings, and conclusions do not reflect the views of the funding agencies, cooperating institutions, or other individuals. 


\section{References}

1. Williamson Shaffer D (2018) Big Data for Thick Description of Deep Learning. In: Millis K, Long D, Magliano J, Wiemer K (eds) Deep Comprehension: Multi-Disciplinary Approaches to Understanding, Enhancing, and Measuring Comprehension, 1st ed. Routledge, pp 262-275.

2. Williamson Shaffer D (2017) Quantitative Ethnography. Cathcart Press.

3. Cai Z, Siebert-Evenstone AL, Eagan B, Williamson Shaffer D, Hu X, Graesser A (2019) nCodert: A Semantic Tool for Improving Recall of nCoder Coding. In: Advances in Quantitative Ethnography. Springer, Madison, WI, pp 41-54.

4. Bakharia A (2019) On the Equivalence of Inductive Content Analysis and Topic Modeling. In: Advances in Quantitative Ethnography. Springer, Madison, WI, pp 291-298.

5. Eagan B, Swiecki Z, Farrell C, Williamson Shaffer D (2019) The Binary Replicate Test: Determining the Sensitivity of CSCL Models to Coding Error. In: Wide Lens: Combining Embodied, Enactive, Extended, and Embedded Learning in Collaborative Settings. International Society of the Learning Sciences, Lyon, France, pp 328-335.

6. Gee J (2014) An Introduction to Discourse Analysis: Theory and Method. Routledge, London.

7. Ruis AR, Siebert-Evenstone AL, Pozen R, Eagan BR, Williamson Shaffer D (2019) Finding Common Ground: A Method for Measuring Recent Temporal Context in Analyses of Complex, Collaborative Thinking. In: A Wide Lens: Combining Embodied, Enactive, Extended, and Embedded Learning in Collaborative Settings. International Society of The Learning Sciences, Lyon, France, pp 136-143.

8. Wooldridge A, Haefli R (2019) Using Epistemic Network Analysis to Explore Outcomes of Care Transitions. In: Eagan B, Misfeldt M, Siebert-Evenstone AL (eds) Advances in Quantitative Ethnography. Springer, Madison, WI, pp 245-256.

9. Frank K, Min K (2007) Indices of Robustness for Sample Representation. Sociological Methodology 37:349-392.

10. Zörgő S, Peters G (2019) Epistemic Network Analysis for Semi-Structured Interviews and Other Continuous Narratives: Challenges and Insights. In: Advances in Quantitative Ethnography., Eagan B., Misfeldt M., Siebert-Evenstone A. (eds). Springer Nature, Switzerland, pp 267-277.

11. Swiecki Z (2020). Modeling interdependence in collaborative problem-solving [Doctoral dissertation, University of Wisconsin-Madison]. 